\title{
The aging lung: tissue telomere shortening in health and disease
}

Stephanie Everaerts ${ }^{1 * \dagger}$ (D), Elise J. Lammertyn ${ }^{1 \dagger}$, Dries S. Martens ${ }^{2}$, Laurens J. De Sadeleer ${ }^{1}$, Karen Maes $^{1}$, Aernoud A. van Batenburg ${ }^{3}$, Roel Goldschmeding ${ }^{4}$, Coline H. M. van Moorsel ${ }^{3,5}$, Lieven J. Dupont ${ }^{1,6}$, Wim A. Wuyts ${ }^{1,6}$, Robin Vos ${ }^{1,6}$, Ghislaine Gayan-Ramirez ${ }^{1}$, Naftali Kaminski ${ }^{7}$, James C. Hogg ${ }^{8}$, Wim Janssens ${ }^{1,6}$, Geert M. Verleden ${ }^{1,6}$, Tim S. Nawrot ${ }^{2,9}$, Stijn E. Verleden' ${ }^{1}$, John E. McDonough ${ }^{1}$ and Bart M. Vanaudenaerde ${ }^{1}$

\begin{abstract}
Background: Telomere shortening has been associated with several lung diseases. However, telomere length is generally measured in peripheral blood leucocytes rather than in lung tissue, where disease occurs. Consequently, telomere dynamics have not been established for the normal human lung nor for diseased lung tissue. We hypothesized an age- and disease-dependent shortening of lung tissue telomeres.
\end{abstract}

Methods: At time of (re-)transplantation or autopsy, 70 explant lungs were collected: from unused donors (normal, $n=13$ ) and patients with cystic fibrosis (CF, $n=12$ ), chronic obstructive pulmonary disease (COPD, $n=11)$, chronic hypersensitivity pneumonitis ( $\mathrm{CHP}, n=9)$, bronchiolitis obliterans syndrome $(\mathrm{BOS})$ after prior transplantation $(n=11)$ and restrictive allograft syndrome (RAS) after prior transplantation $(n=14)$. Lungs were inflated, frozen and then scanned using CT. Four tissue cores from distinct lung regions were sampled for analysis. Disease severity was evaluated using $C T$ and micro $C T$ imaging. DNA was extracted from the samples and average relative telomere length (RTL) was determined using real-time qPCR.

Results: The normal lungs showed a decrease in RTL with age $(p<0.0001)$. Of the diseased lungs, only BOS and RAS showed significant RTL decrease with increasing lung age ( $p=0.0220$ and $p=0.0272$ respectively).

Furthermore, we found that RTL showed considerable variability between samples within both normal and diseased lungs. CHP, BOS and RAS lungs had significant shorter RTL in comparison with normal lungs, after adjustment for lung age, sex and BMI ( $p<0.0001, p=0.0051$ and $p=0.0301$ respectively). When investigating the relation between RTL and regional disease severity in CF, CHP and RAS, no association was found.

Conclusion: These results show a progressive decline in telomere length with age in normal, BOS and RAS lungs. CHP, BOS and RAS lungs demonstrated shorter RTL compared to normal lungs. Lung tissue RTL does not associate with regional disease severity within the lung. Therefore, tissue RTL does not seem to fully reflect peripheral blood telomere length.

Keywords: Cystic fibrosis, Chronic obstructive pulmonary disease, Chronic hypersensitivity pneumonitis, Chronic lung allograft dysfunction, BOS, RAS, Cellular senescence, Telomere length

\footnotetext{
* Correspondence: stephanie.everaerts@kuleuven.be

'Equal contributors

${ }^{1}$ Laboratory of Respiratory Diseases, Department of Chronic Diseases,

Metabolism \& Aging (CHROMETA), KU Leuven, Herestraat 49, O\&NI, box 706,

B-3000 Leuven, Belgium

Full list of author information is available at the end of the article
}

(c) The Author(s). 2018 Open Access This article is distributed under the terms of the Creative Commons Attribution 4.0 International License (http://creativecommons.org/licenses/by/4.0/), which permits unrestricted use, distribution, and reproduction in any medium, provided you give appropriate credit to the original author(s) and the source, provide a link to the Creative Commons license, and indicate if changes were made. The Creative Commons Public Domain Dedication waiver (http://creativecommons.org/publicdomain/zero/1.0/) applies to the data made available in this article, unless otherwise stated. 


\section{Background}

Aging is a complex biological process characterized by progressive decline of all physiological functions resulting in a time-dependent increase in mortality $[1,2]$. With increasing age, the respiratory system undergoes structural remodelling, including rearrangement of extracellular matrix, dilatation of alveoli and enlargement of airspaces leading to lung function decline. This process of structural aging together with immunosenescence leads to an increased susceptibility to acute and chronic pulmonary disease, which can be influenced by individual factors, such as genetic background and exposure $[3,4]$.

One of the defining mechanisms behind aging is related to telomere shortening $[5,6]$. Telomeres are stretches of repetitive DNA capping the ends of chromosomes, protecting them from unscheduled DNA degradation. In somatic cells, telomeres undergo attrition at each cell replication until the point they become dysfunctional, inducing a DNA damage response leading to cellular senescence. Shortened peripheral blood leukocyte telomeres have been associated with various age-related diseases, such as atherosclerosis [7] and type 2 diabetes mellitus [8], and an increased risk for some cancers [9]. This suggests that the increased turnover and replication of circulating leukocytes during chronic inflammation accelerates the rate of leukocyte telomere shortening $[7,10]$.

In patients with respiratory disorders such as chronic obstructive pulmonary disease (COPD) and interstitial lung disease (ILD), shorter peripheral blood leukocyte telomeres have been demonstrated compared to healthy individuals [11-13]. Moreover, mutations in essential telomerase genes, telomerase reverse transcriptase (TERT) and RNA template (TR), are associated with idiopathic pulmonary fibrosis (IPF) and COPD [14, 15]. Furthermore, a single-nucleotide polymorphism of MUC5B, which is associated with both IPF and chronic hypersensitivity pneumonitis (cHP), associates with shorter blood leucocyte telomere length in cHP [16]. However, much less is known about telomere length within the lung tissue, where cell turnover is low compared to blood leucocytes [17]. Moreover, telomere dynamics in healthy aging lung have not been established.

We hypothesized an age- and disease-dependent shortening of lung tissue telomeres, as has been shown in blood leucocyte telomeres. Explanted lung tissue was used, based on the availability in our lung transplant center. First, relative telomere length (RTL) in unused donor lungs was measured to determine telomere length in normal human lung tissue. Second, RTL was measured in lung tissue of patients suffering from end-stage chronic lung diseases including COPD, cHP, cystic fibrosis (CF) and two phenotypes of chronic lung allograft dysfunction (CLAD) after prior lung transplantation. Finally, we investigated the association between regional disease severity within the lung and RTL.

\section{Methods \\ Study material}

Based on the availability in our lung transplant center, an assortment of 70 explant lungs was collected between 2009 and 2015, including 13 unused donor lungs, which were used to determine telomere length in normal human lung tissue. These lungs were obtained after decline for lung transplantation (LTx) by the handling surgeon due to persistent (micro) thrombi despite flushing $(n=4)$, unexpected death of the recipient $(n=1)$, mild contusion $(n=2)$, presence of a kidney tumour in the donor $(n=2)$, suspicion of emphysema $(\mathrm{n}=1)$, beginning fibrosis $(\mathrm{n}=1)$ , infection $(n=1)$ or rupture of an artery $(n=1)$. Two donor lungs of contrasting age, which were declined because of edema and infection, were collected for an additional experiment. According to Belgian law, organs from prospective donors, which are of insufficient quality for LTx and have been conclusively declined by the transplant surgeon, can be used for research purposes. CF $(n=12)$, COPD $(n=11)$ and cHP $(n=9)$ explant lungs were collected at the time of LTx. CLAD lungs $(n=25)$, subdivided into bronchiolitis obliterans syndrome (BOS) $(n=11)$ and restrictive allograft syndrome (RAS) $(n=14)$, were collected during re-LTx or autopsy. Diagnostic criteria are listed in Additional file 1. All LTx patients gave written informed consent to use their lungs for research purposes. The study was approved by the Medical Ethics Board of University Hospitals Leuven, Belgium (ML6385).

\section{Lung tissue processing}

All lungs were processed as previously described [18, 19]. In brief, the main stem bronchus was cannulated and lungs were inflated to near total lung capacity, maintained at $10 \mathrm{~cm}$ water pressure and subsequently frozen in liquid nitrogen vapours before storing at $-80^{\circ}$ C. High resolution computed tomography (HRCT) scan was taken of all frozen lungs. Lungs were cut into $2 \mathrm{~cm}$ thick slices from apex to base with cores (diameter 1 . $4 \mathrm{~cm}$ ) systematically removed using a drill press. In this study, four cores per lung were analysed: two cores from apical slices (slice number 2 to 5 ) and two cores from basal slices (slice number 6 to 12), randomly selected to reflect the spatial heterogeneity within the lung. In CF, an additional distinction was made between cores taken from an area with structural abnormalities including bronchiectasis, airway destruction and increased tissue density on HRCT, and normal-appearing tissue. Tissue of unused donor lungs was processed in the same way, except that areas with suspicion of any abnormality were strictly avoided while sampling. 


\section{Micro CT analysis}

Micro CT scanning was used to obtain high-resolution images of lung tissue and measure disease severity in the cores. Frozen cores were scanned using a Bruker Skyscan 1172 micro CT device (Bruker, Kontich, Belgium) with a resolution of $10 \mu \mathrm{m}$ while maintained at $-30{ }^{\circ} \mathrm{C}$, using a cooling stage. Scans were reconstructed using NRecon and images analysed using CTAn software (Bruker). Normal lung parenchyma with a well-developed alveolar structure has a high surface area to volume ratio (surface density). Consequently, measurement of surface density was used to determine the extent of normal tissue within each sample with decreasing values reflecting loss of normal tissue through disease (e.g. emphysema in COPD or fibrosis in cHP/RAS). Cores of RAS and cHP lungs were stratified based on the median surface density value of their disease group.

\section{Relative telomere length measurement}

DNA was extracted from a portion of each lung tissue core (height: $0.5 \mathrm{~cm}$, diameter: $0.7 \mathrm{~cm}$ ) using the QIAamp DNA Mini Kit (Qiagen Inc., Venlo, The Netherlands). DNA concentration and purity were determined using NanoDrop (Thermo Scientific NanoDrop Technologies, Wilmington, Delaware, USA) and DNA integrity was checked using agarose gel electrophoresis. Average RTL was measured using a modified quantitative real-time PCR (qPCR) protocol as described previously [20]. Details are provided in Additional file 1.

\section{Fluorenscent in situ hybridization}

An extensive description of the processing for fluorescent in situ hybridization (FISH) is provided in Additional file 1. Telomere labelling was performed on tissue slices using a telomere-Cy3 PNA Probe (Panagene, Daejeon, South-Korea). Alveolar type 2 (AT2) cells were labelled with pro-SPC staining (AB3786,1/500,Merck Millipore, Darmstadt, Germany), DNA of the tissue slides was stained using 4',6-diamidino- 2-phenylindole (DAPI, $25 \mu \mathrm{g} / \mathrm{mL}$ ).

A Fluorescence microscope (Leica DM 5500B) at high magnification $(63 \times)$ was used for image capture. Multiple images per slice were taken within $24 \mathrm{~h}$ after staining, all pro-SPC positive cells were used. Mean relative telomere signal per cell was calculated as the total Cy3 area divided by the DAPI signal per cell.

\section{Statistical analyses}

All statistical analyses were performed using SAS 9.4 (SAS Institute Inc., Cary, NC, USA) and R 3.2.2 statistical software ( $\mathrm{R}$ Foundation for Statistical Computing, Vienna, Austria). Graphical representation of data was generated using GraphPad Prism 4.0 Software (GraphPad Software, San Diego, CA, USA) and R 3.2.2. Prior to analyses, RTL of each core was $\log _{10}$ transformed to normalize the dataset. Demographic and clinical characteristics were compared by Kruskal-Wallis analysis with Dunn's correction for multiple testing in case of continuous variables and $\mathrm{Chi}^{2}$ for discrete variables. The association of tissue core RTL with lung age was analysed by mixed linear models with the original lung as random effect and adjustment for BMI and sex. Paired t-test analysis was used to investigate regional differences within the groups. RTL comparison between specific diseases and normal lungs was performed with a mixed linear model with the original lung as random effect, adjusted for lung age, sex and BMI. An unpaired t-test was performed to compare tissue cores with normal versus abnormal appearance (in CF) and mild versus severe disease (in cHP and RAS). A mixed model with the original lung as random effect was used to assess the association between core RTL and surface density, while accounting for lung age, BMI and sex. The coefficient of variation $(\mathrm{CV})$ was calculated per disease group (inter-lung variability) and within the lungs (intra-lung variability). $P$-values $<0.05$ were considered significant in all analyses.

\section{Results}

\section{Patient characteristics}

Demographic and clinical characteristics are summarized in Table 1. Lung age reflects the calendar age of the transplanted subject or the lung donor in case of CLAD. Lung function of the normal lungs was unknown. CF lung age was significantly lower than age of normal lungs. Patients with CF, BOS or RAS had a significantly lower BMI compared to donors of unused normal lungs. Other diseases showed no significant differences compared to characteristics of normal lungs.

\section{Relative telomere length in normal lung tissue}

Unused donors were between 16 and 72 years of age. Each 1 -year increase in age was associated with a significant decrease in RTL $(p<0.0001)$ (Table 2). Figure 1a and Table 3 show the inter- and intra-lung variance in RTL for normal lungs (CV of 18.3 and $16.3 \%$ respectively). RTL of cores originating from apical lung slices was longer compared to basal cores $(p=0.0002)$ (Fig. 1b). Also, when considering lobar distribution, upper lobe tissue had longer RTL compared to lower lobe tissue ( $p=0.0015)$ (Fig. 1c).

In order to confirm the association between age and lung tissue telomere length, we performed FISH on two additionally collected donor lungs of opposite age (19 versus 83 years). Results show a clearly higher telomere length in AT2 cells of the youngest donor $(p=0.009)$ (Fig. 2).

\section{Relative telomere length in lung disease}

The range of lung age was dependent on underlying disease (Table 1). The relation between lung tissue RTL and 
Table 1 Demographic and clinical characteristics of lungs

\begin{tabular}{|c|c|c|c|c|c|c|}
\hline & NORMAL & CF & COPD & $\mathrm{cHP}$ & BOS & RAS \\
\hline Subjects, n & 13 & 12 & 11 & 9 & 11 & 14 \\
\hline Lung age, years & $48(20)$ & $23(7)^{*}$ & $60(3)$ & $58(10)$ & $27(24)$ & $46(23)$ \\
\hline Lung age, range & $16-72$ & $19-33$ & $48-61$ & $36-61$ & $16-48$ & $9-61$ \\
\hline Male, n (\%) & $10(77)$ & $5(42)$ & $5(45)$ & $4(44)$ & $5(45)$ & $8(57)$ \\
\hline Male donor, n (\%) & NA & NA & NA & NA & $5(45)$ & $8(57)$ \\
\hline Patient height, $\mathrm{cm}$ & $175(15)$ & $162(18)$ & $163(22)$ & $166(19)$ & $168(12)$ & $168(17)$ \\
\hline Patient weight, kg & $80(25)$ & $46(14)^{* * *}$ & $60(17)$ & $74(9)$ & $51(21)^{* *}$ & $60(21)^{*}$ \\
\hline $\mathrm{BMI}, \mathrm{kg} / \mathrm{m}^{2}$ & $25(4)$ & $18(2)^{* * *}$ & $21(8)$ & $27(4)$ & $18(5)^{* *}$ & $19(6)^{* *}$ \\
\hline $\mathrm{FEV}_{1}, \mathrm{~L}$ & NA & $0.8(0.4)$ & $0.8(0.5)$ & $1.1(0.8)$ & $0.6(0.3)$ & $0.9(0.5)$ \\
\hline $\mathrm{FEV}_{1}, \%$ predicted & NA & $23(10)$ & $31(11)$ & $49(21)$ & $20(3)$ & $26(12)$ \\
\hline FVC, L & NA & $1.6(0.8)$ & $2.0(0.4)$ & $1.3(0.9)$ & $1.5(1)$ & $1.4(0.6)$ \\
\hline FVC, \% predicted & NA & $45(15)$ & $66(33)$ & $38(20)$ & $46(20)$ & $33(13)$ \\
\hline $\mathrm{FEV}_{1} / \mathrm{FVC}$ & NA & $0.5(0.1)$ & $0.4(0.1)$ & $0.9(0.2)$ & $0.4(0.1)$ & $0.7(0.3)$ \\
\hline DLco, \% predicted & NA & $38(33)^{\mathrm{a}}$ & $33(14)$ & $30(7)$ & $40(18)^{b}$ & $35(8)^{c}$ \\
\hline
\end{tabular}

a, b, c respectively 6, 1 and 7 missing values. Results are given as $n$ (\%) or median (IQR). \% predicted of FEV1 and FVC was based on ECSC equations before 2012 [45] and on GLI equations from 2012 onwards [46]. ATS recommendations were used for equations of DLCO reference [47]. Significant difference with the normal lungs is indicated with ${ }^{*} p<0.05,{ }^{* *} p<0.01$ and ${ }^{* * *} p<0.001$

CF cystic fibrosis, COPD chronic obstructive pulmonary disease, CHP chronic hypersensitivity pneumonitis, BOS bronchiolitis obliterans syndrome, RAS restrictive allograft syndrome, NA not applicable, BMI body mass index, FEV1 forced expiratory volume in $1 \mathrm{~s}, F V C$ forced vital capacity, FEV1/FVC tiffeneau index, DLco diffusion capacity of the lung for carbon monoxide

age is shown per disease in Table 2. In BOS and RAS, RTL decreased significantly with every 1 -year increase in donor lung age ( $p=0.022$ and $p=0.027$ respectively). This is in contrast with COPD, where a significant increase in RTL was observed with every 1-year increase in age $(p=0.0016)$.

When affected lung tissue was compared with normal lung tissue, RTL was $-28.0 \%$ shorter in cHP tissue $(\mathrm{p}<0.0001),-14.6 \%$ shorter in BOS tissue $(p=0.0051)$ and $-10.4 \%$ shorter in RAS tissue $(p=0.030)$ in an age, sex and BMI-adjusted model. CF and COPD tissue was

Table 2 Relation between RTL and lung age in normal and diseased lung tissue

\begin{tabular}{lllll}
\hline Group & Estimate & \% change & $95 \% \mathrm{Cl}$ & $p$-value \\
\hline NORMAL & -0.0039 & -0.90 & $-1.26--0.54$ & $\mathbf{0 . 0 0 0 1}$ \\
CF & -0.0045 & -1.04 & $-2.21-0.16$ & 0.090 \\
COPD & 0.015 & 3.41 & $1.36-5.49$ & $\mathbf{0 . 0 0 1 6}$ \\
CHP & -0.0024 & -0.55 & $-4.98-4.10$ & 0.81 \\
BOS & -0.0033 & -0.76 & $-1.41--1.41$ & $\mathbf{0 . 0 2 2}$ \\
RAS & -0.0021 & -0.47 & $-0.88--0.06$ & $\mathbf{0 . 0 2 7}$ \\
\hline
\end{tabular}

Mixed linear models were used to measure the association between tissue RTL and lung age, with the lung as random effect, adjusted for sex and BMI. Estimates are presented as percentage change $(95 \% \mathrm{Cl})$ in average RTL for each 1-year increase in lung age

RTL relative telomere length, 95\% Cl 95\% confidence interval, CF cystic fibrosis, COPD chronic obstructive pulmonary disease, $C H P$ chronic hypersensitivity pneumonitis, $B O S$ bronchiolitis obliterans syndrome, RAS restrictive allograft syndrome

$p$-value $<0.05$ is captured in bold not different from normal lung tissue in the same model (Table 4).

Figure 3a-e and Table 3 show that in diseased lungs, RTL had considerable variability, both between lungs and within the same lung. A difference in RTL between regions or lobes could not be demonstrated in the diseased lungs, as was observed in the normal lungs (Additional file 1).

\section{Relative telomere length, structural abnormalities and disease severity}

Given the low variability in surface density of cores from normal, COPD and BOS lungs, the association between RTL and regional disease severity was not investigated in these lungs. In CF, there was no difference in RTL between tissue cores originating from areas with structural abnormalities on HRCT or normal-appearing tissue (both $n=24)(p=0.96)$ (Fig. 4a). In cHP, RTL did not differ between mildly and severely diseased tissue cores (both $n=18)(p=0.22)$ (Fig. 4b). In RAS, severely diseased tissue cores tended to have longer RTL compared to mildly affected cores (both $n=28)(p=0.084)$ (Fig. 4c). In addition, multivariate analysis demonstrated that for every decrease in surface density of $0.01 / \mu \mathrm{m}$ in RAS tissue, there was an increase in RTL of $1.00 \%$ (95\% CI: -1.00 to -0.99 , $p=0.0027$ ) while accounting for lung age, sex and BMI. The same model could not reveal a significant association between RTL and surface density in CF nor in cHP. 

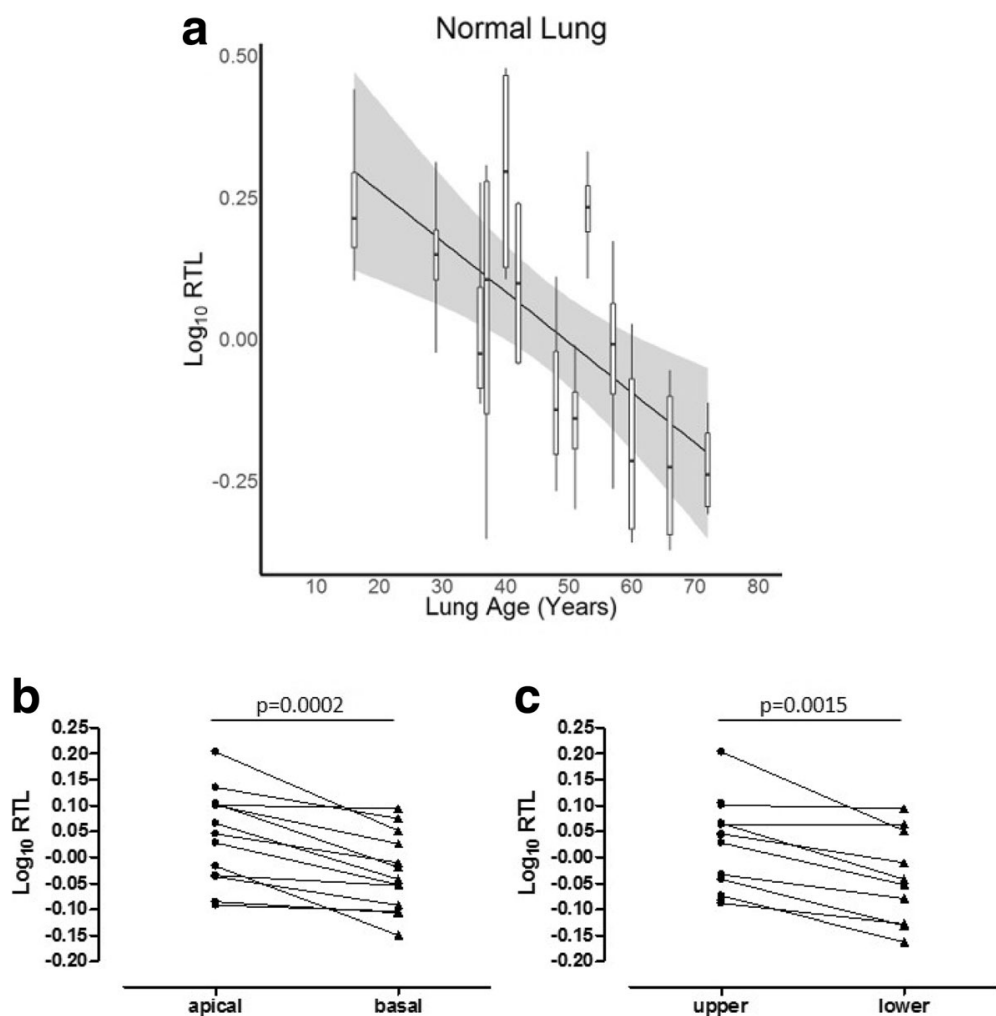

Fig. 1 RTL decrease with age and regional difference in normal lung tissue. a: $\log _{10}$ RTL versus lung age in normal lungs. Log $\log _{10}$ RTL per lung is presented as a boxplot with the grey area representing the $95 \%$ confidence interval. $\mathbf{b}$ and $\mathbf{c}$ : Paired t-test of $\log _{10}$ RTL in normal lungs based on spatial distribution. Every dot shows the mean $\log _{10}$ RTL of cores originating from the respective region per lung. $\mathbf{b}$ : difference in $\log _{10}$ RTL between cores originating from the apical $(n=26)$ and basal $(n=26)$ lung regions $(p=0.0002)$. c: difference in $\log _{10}$ RTL between upper $(n=37)$ and lower lobe $(n=15)$ cores $(p=0.0015)$

\section{Discussion}

The present study demonstrated an age-dependent RTL decline in normal human lung tissue. In disease-affected lung tissues, the association between lung age and shorter RTL was only present in both CLAD phenotypes. CHP, BOS and RAS tissue had significantly shorter tissue RTL in comparison with normal lungs, when accounting for lung age, BMI and sex. RTL appeared to vary within the studied diseases and within the lung, however we could not demonstrate a link between regional disease severity and shorter tissue RTL.

The association between increasing age and telomere shortening in highly proliferative tissues, such as peripheral blood leucocytes, has been established for quite some time now [21], but knowledge on age-dependent telomere attrition in lung tissue remains scarce. Daniali et al. have shown that there is large variability in telomere length in somatic tissues of the body, which is predominantly established early in life [22, 23]. Highly proliferative tissues, such as blood and skin, have shorter telomeres than minimally proliferative tissues, such as muscle and fat. Nevertheless, it was demonstrated that rates of telomere shortening are similar with increasing age and that telomere length is highly correlated between these tissues [22]. Moreover, a study in primates demonstrated shorter lung telomeres in older animals compared to young ones [24]. Our data confirmed that telomere length does shorten with increasing age in the normal human lung.

Table 3 Coefficients of variation (\%) for RTL between and within lungs

\begin{tabular}{|c|c|c|c|c|c|c|c|}
\hline & NORMAL & CF & COPD & $\mathrm{CHP}$ & BOS & RAS & Mean \\
\hline CV between lungs for each group, $\%$ & 18.3 & 12.5 & 17.6 & 30 & 22.4 & 18.0 & 19.8 \\
\hline Mean CV within lung, \% & 16.3 & 11.2 & 17.3 & 15.2 & 11.9 & 12.4 & 14.1 \\
\hline
\end{tabular}

Results are presented as percentages. Coefficients of variation were calculated per disease group (based on mean lung RTL values) and per lung (based on core RTL values)

RTL relative telomere length, $C V$ coefficient of variation, $C F$ cystic fibrosis, COPD chronic obstructive pulmonary disease, cHP chronic hypersensitivity pneumonitis, $B O S$ bronchiolitis obliterans syndrome, RAS restrictive allograft syndrome 

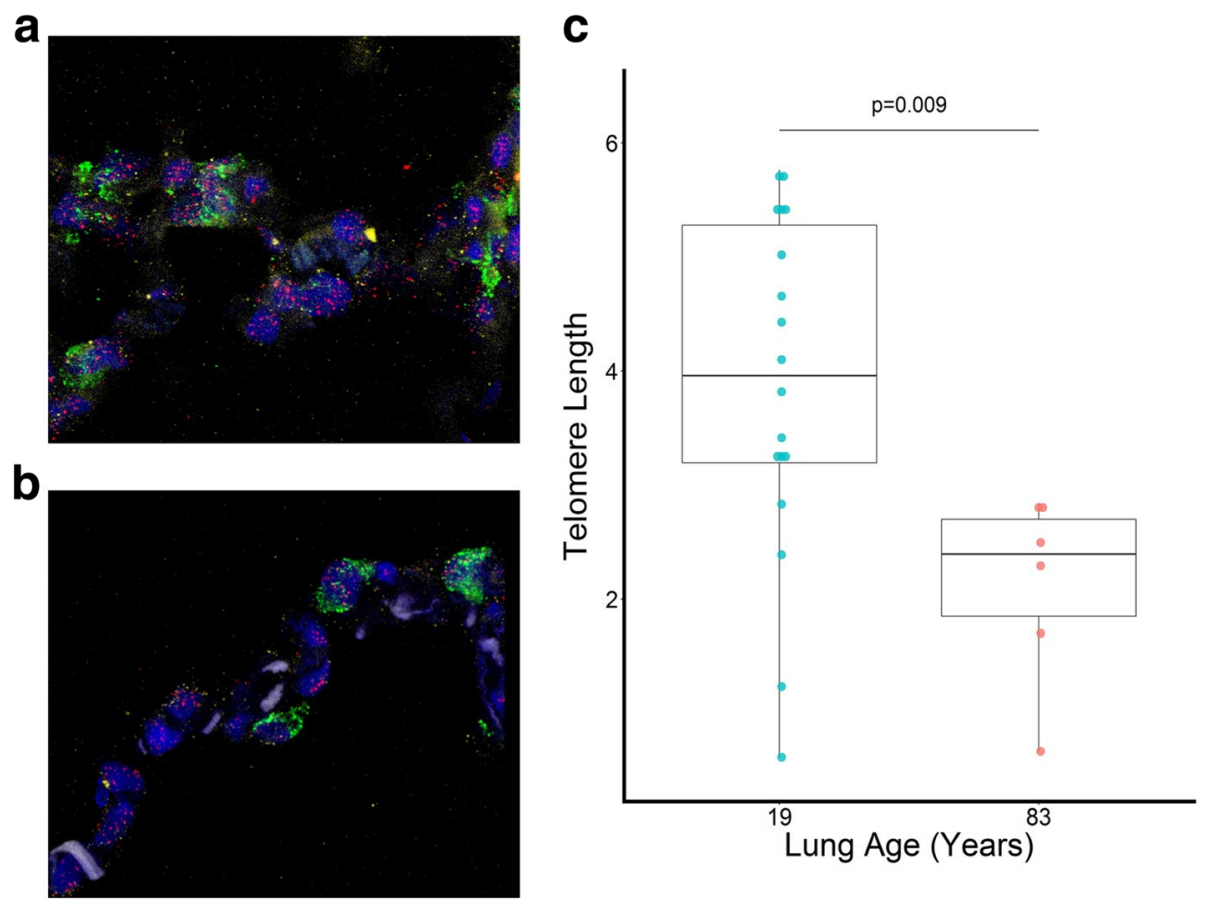

Fig. 2 Association of age and telomere length in AT2 cells of normal lung tissue by fluorescent in situ hybridization. Telomere length determination by FISH on normal lung tissue of (a) a 19-year old and (b) an 83-year old donor showed (c) significantly higher telomere length in AT2 cells of the youngest subject ( $p=0.009$ ). The fluorecent labelling in the $\mathbf{a}$ and $\mathbf{b}$ panel stands for green: proSPC (AT2 cells), red: telomere probe, blue: DAPI. Data are presented as boxplots and every dot represents one cell

Critical telomere shortening due to increasing age or disease may result in cellular senescence [4]. Alveolar type 1 (AT1) and AT2 cells are the most abundant epithelial cells in the lung parenchyma. AT2 cells not only produce surfactant but also serve as progenitor cells for the air-blood barrier forming AT1 cells [4]. Consequently, AT2 cell senescence may explain some of the age-related changes observed in the lungs. In the elderly, an important decline in alveolar epithelial stem cell

Table 4 Comparison between RTL in diseased and normal lungs, adjusted for lung age, BMI and sex

\begin{tabular}{lllll}
\hline Comparison & Estimate & $\%$ change & $95 \% \mathrm{Cl}$ & $p$-value \\
\hline CF vs normal & 0.0090 & 2.09 & $-9.20-14.77$ & 0.73 \\
COPD vs normal & 0.032 & 7.67 & $-2.93-19.44$ & 0.16 \\
CHP vs normal & -0.14 & -27.97 & $-34.63--20.64$ & $<\mathbf{0 . 0 0 0 1}$ \\
BOS vs normal & -0.069 & -14.62 & $-23.53--4.69$ & $\mathbf{0 . 0 0 5 1}$ \\
RAS vs normal & -0.048 & -10.43 & $-18.91--1.06$ & $\mathbf{0 . 0 3 0}$ \\
\hline
\end{tabular}

Multivariate mixed linear model comparing diseased lungs with normal lungs with lung as random effect, adjusted for lung age, BMI and sex. Estimates are presented as percentage change $(95 \% \mathrm{Cl})$ in average RTL per group compared to normal lungs

RTL relative telomere length, 95\% Cl 95\% confidence interval, CF cystic fibrosis, COPD chronic obstructive pulmonary disease, $C H P$ chronic hypersensitivity pneumonitis, BOS bronchiolitis obliterans syndrome, RAS restrictive allograft syndrome, vs versus

$p$-value $<0.05$ is captured in bold renewal, compromising the tissue's regenerative potential, may be observed $[25,26]$, as well as a proinflammatory pulmonary environment maintained by senescent cells via paracrine mediators and promoting further senescence $[1,26]$. Finally, alveolar stem cell exhaustion has been discussed as a key factor in agerelated pulmonary diseases $[27,28]$.

In contrast to the findings in normal and CLAD lungs, we could not demonstrate lung age-related RTL decline in $\mathrm{CF}$ and $\mathrm{cHP}$ lungs. In COPD, the association between tissue RTL and age was even significantly opposite. A few reasons may explain why the association between lung tissue RTL and lung age was not present in each investigated disease. Firstly, the age range was different in each group and was clearly wider in normal, BOS and RAS lungs. Moreover, also disease and host-related factors, other than age, may influence telomere dynamics in the lung. Namely, the activity of telomerase or the expression of essential telomerase genes may significantly determine telomere length. The difference in lung RTL along the apical to basal axis in normal lungs may be explained by the difference in ventilation and perfusion of these regions, with more perfusion in basal regions. Moreover, shorter RTL in basal segments may reflect higher cell turnover, caused by mild atelectasis-related injury, as has been postulated as a mechanism for basal 


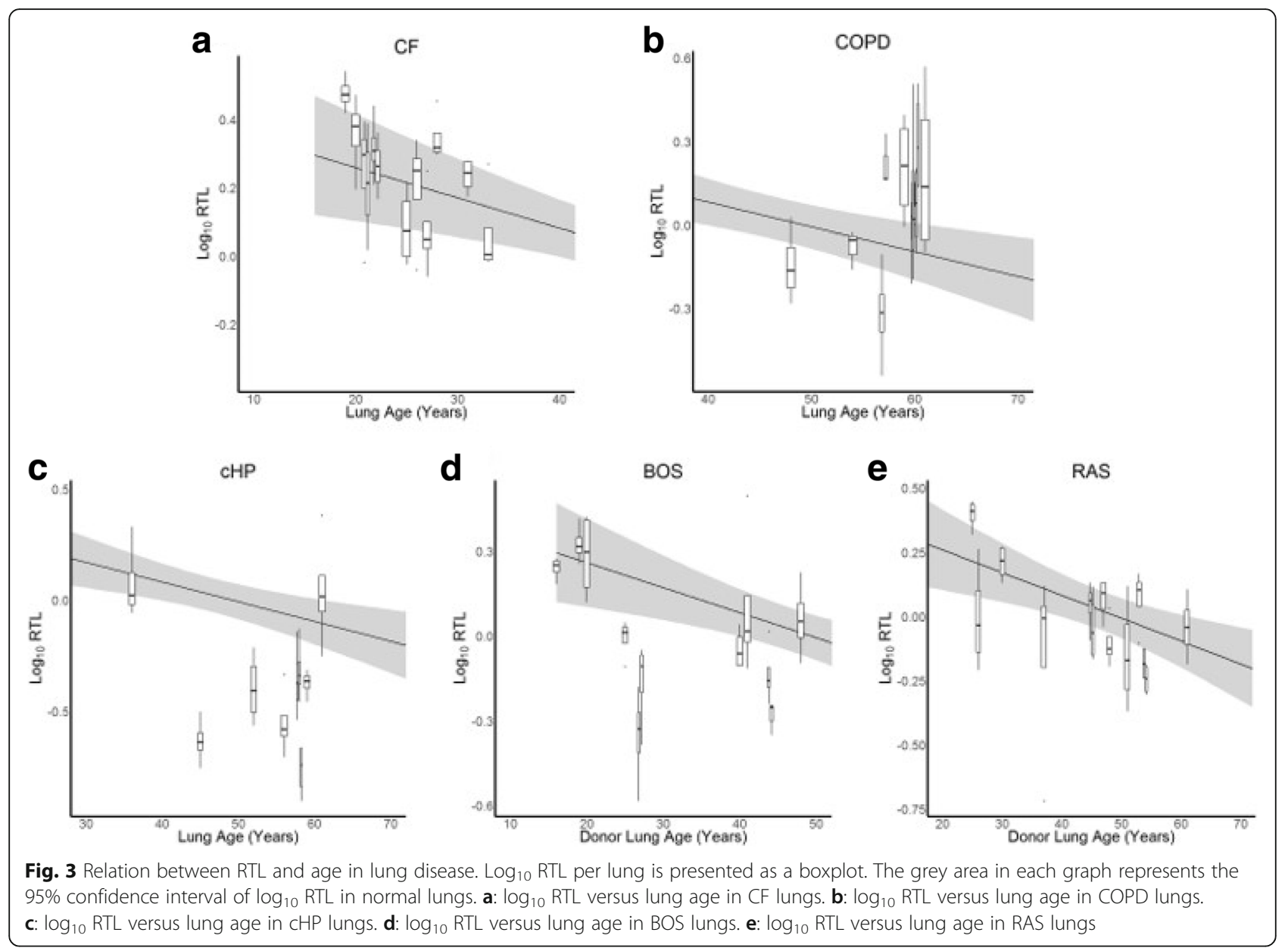

predominance of usual interstitial pneumonia [29]. However, this difference in apical versus basal RTL was not present in diseased lungs.

In $\mathrm{CF}$, little is known on telomere biology to date. Fischer et al. found no difference in telomere length in epithelial cells of CF patients compared to controls [30]. With a fulladjusted model, we could also not demonstrate a shorter RTL in CF compared to normal lungs. Previous studies reported shorter peripheral blood LTL in COPD [11, 31, 32 ] and telomerase mutations have been demonstrated in $1 \%$ of COPD patients, which was associated with emphysema in families with autosomal dominant telomeremediated disease including pulmonary fibrosis [15]. Studies investigating telomere length of COPD airway epithelial cells are less conclusive. Tsuji et al. found that telomere length in AT2 cells was significantly shorter in patients with

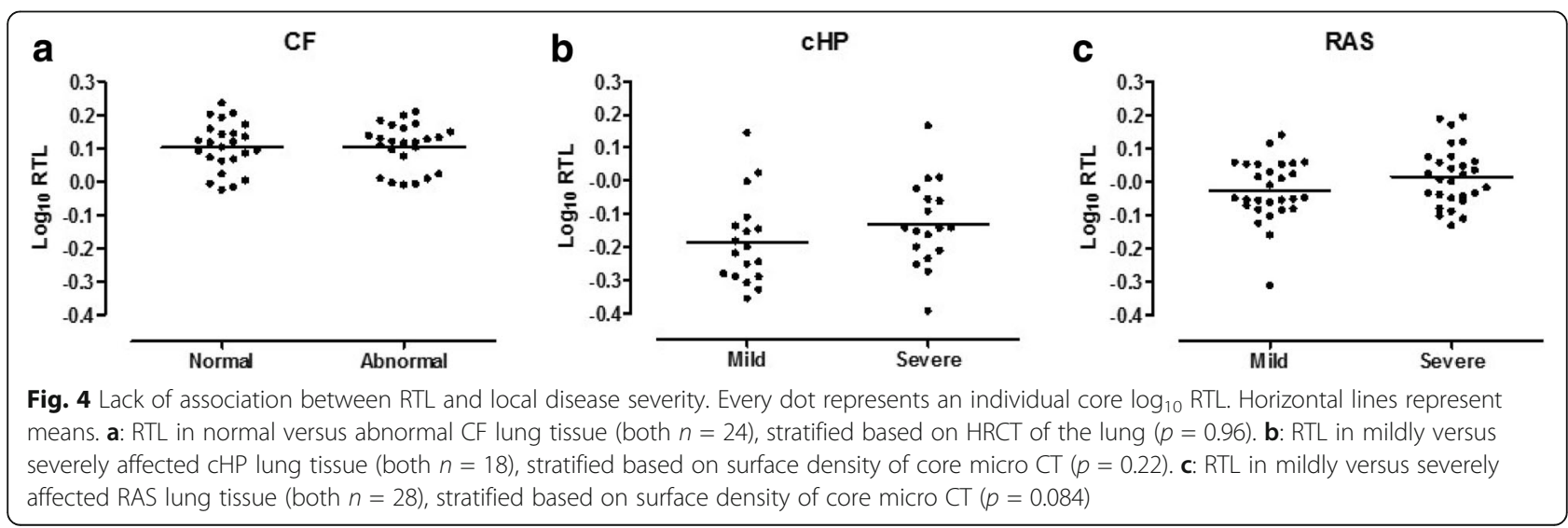


emphysema compared to non-smokers [33], whereas Birch and colleagues could not demonstrate shorter telomeres in cultured, primary small airway epithelial cells isolated from COPD patients compared to age-matched controls [34]. The results of our study demonstrate even longer tissue RTL in COPD lungs compared to normal lungs. This finding is surprising, although accelerated aging in COPD may be rather explained by other mechanisms than telomere shortening, such as impairment of anti-aging molecules due to oxidative stress [35]. Furthermore, we are aware that patients with COPD that are eligible for transplantation, represent a subgroup with less comorbidities and possibly less systemic inflammation.

In CLAD, telomere dysfunction is increasingly appreciated as a research target. A small study in lung transplant patients found no association of donor peripheral blood LTL or recipient tissue RTL with survival [36]. More recently, Faust et al. reported that short donor peripheral blood LTL was associated with worse CLAD-free survival after transplantation. In addition, lung allografts later progressing to CLAD demonstrated shorter telomeres in endobronchial biopsies taken within the first 90 days post LTx, suggesting that decreased telomere length within the lung may contribute to CLAD [37]. Our results, showing shorter RTL in CLAD versus normal lungs, support this finding in whole-lung tissue. Within CLAD, RTL did not allow us to differentiate between both phenotypes.

Next to BOS and RAS, also cHP lungs exhibited shorter RTL compared to normal lungs. Idiopathic pulmonary fibrosis (IPF), another interstitial lung disease, is the most frequent manifestation of telomeraseassociated disease [14] and shorter peripheral blood LTL has been reported in this disease [12]. In families with multiple pulmonary fibrosis patients, IPF or cHP may occur, suggesting that these disorders have common risk factors. Assuming genetic and clinical analogies between cHP and IPF [16], telomere dysfunction may play a bigger role in the pathophysiology of cHP compared to the other end-stage diseases we investigated.

Despite the reported associations of shorter peripheral blood LTL and increased mortality in CHP [16] and CLAD [37], and the association between shorter telomeres and the extent of fibrosis in both peripheral blood LTL of cHP patients [16] and AT2 cells of IPF patients [38], we could not show an association between lung tissue RTL and local disease severity in cHP and RAS. For cHP, the explanation may be that shorter telomeres encompass a sensitivity for other harmful hits required to manifest disease, as has been suggested for telomere-mediated diseases [23]. Surprisingly, severely diseased RAS tissue cores had longer RTL compared to mildly diseased tissue independent of lung age, sex and BMI. A possible explanation is that the evolution from a normal lung allograft to RAS goes very fast compared with the disease progression of the other studied pathologies, with graft loss occurring 0. 6-1.5 years after diagnosis [39]. Even though CF lung disease occurs with an upper lobe predominance [40], we did not find shorter RTL in tissue cores originating from the upper lobes, nor did cores coming from areas with $\mathrm{CT}$ alterations demonstrate shorter RTL.

Our results of lung tissue RTL do not fully reflect results from previous studies about peripheral LTL in respiratory diseases. The reason is probably the difference in turnover between these cells. In telomere-mediated diseases, highturnover tissue with shorter telomeres more rapidly lead to telomere dysfunction, causing cellular senescence whereas short telomeres in low-turnover tissue require other genetic or acquired hits to induce telomere dysfunction [23]. Nevertheless, a previous study on telomere length in COPD and $\alpha_{1}$-antitrypsin deficient patients reported a significant correlation between blood and lung tissue telomere length, notwithstanding that blood LTL was significantly shorter compared to lung tissue telomeres, and a healthy control group was lacking [41].

The use of qPCR to measure RTL may be a limitation of this study, although the main advantage is that it is suitable for high-throughput measurements, requiring a small amount of DNA [42]. A high degree of variation is inherent to this technique, due to differential amplification efficiency or variation in measurement between samples [43]. The use of large pieces of lung tissue, representing multiple cell types contributes to the variability since the occurrence of cellular senescence could be tissue- or cell type-specific and telomere shortening may occur at different rates depending on the cell type. Nevertheless, we would like to emphasize that RTL in this study was measured in triplicate with a $\mathrm{T} / \mathrm{S}$ ratio coefficient of variation of $7.4 \%$, which can be considered normal. Quantitative FISH methods are probably more accurate and allow cell-specific measurements, but are not a suitable option to determine telomere length on a large scale. Nevertheless, we applied this technique to normal lungs tissue of opposite age to confirm the association between telomere length and lung age.

Another limitation is the lack of peripheral blood of lung donors and transplanted patients in order to determine LTL. This would have enabled us to compare the individual's LTL with tissue-specific RTL and draw conclusions on a possible correlation between both and their respective value as predictors of disease progression and severity. Unfortunately, this study lacks data of explanted IPF lungs, which is -at least partly- a disease of deficient telomere maintenance [14, 44]. Finally, the lungs included in this study were from patients with end-stage disease that had undergone transplantation, representing a highly specific subgroup of patients. While this precludes drawing any conclusions about early disease stages, any association 
between telomere dysfunction and disease pathology would likely be more pronounced in these severely diseased lungs.

\section{Conclusion}

In conclusion, this is the first study investigating tissue RTL of normal lungs and end-stage CF, COPD, cHP, BOS and RAS lungs. We demonstrated that RTL inversely correlated with lung age in normal, BOS and RAS lungs, was the opposite in COPD, and not correlated in CF. As well, cHP, BOS and RAS lungs had shorter RTL compared to normal lungs, although telomere length was not associated with regional disease severity.

\section{Additional file}

Additional file 1: Supplementary materials, methods and results. (DOCX $21 \mathrm{~kb})$

\section{Abbreviations}

AT1: Alveolar type 1 cell; AT2: Alveolar type 2 cell; BMI: Body mass index; BOS: Bronchiolitis obliterans syndrome; CF: Cystic fibrosis; CHP: Chronic hypersensitivity pneumonitis; CLAD: Chronic lung allograft dysfunction; COPD: Chronic obstructive pulmonary disease; CT: Computed tomography; CV: Coefficient of variation; DAPI: 4',6-diamidino- 2-phenylindolefluorescent; FISH: Fluorescent in situ hybridization; HRCT: High resolution computed tomography; ILD: Interstitial lung disease; IPF: Idiopathic pulmonary fibrosis; IQR: Interquartile range; LTL: Leukocyte telomere length; LTx: Lung transplantation; qPCR: Quantitative polymerase chain reaction; RAS: Restrictive allograft syndrome; RTL: Relative telomere length; SD: Standard deviation; T/S ratio: The ratio of telomere copy number to single-copy gene number

\section{Acknowledgements}

The authors would like to thank Herbert Decaluwe, Paul de Leyn, Philippe Nafteux, Dirk Van Raemdonck and Hans Van Veer for providing explanted lung tissue.

\section{Funding}

This study was supported by a KU Leuven Research Fund (C24/15/030), the Astra Zeneca Chair KU Leuven, the Alphonse and Jean Forton Award of the King Baudouin Foundation and the 7th Framework Programme (FP7) of the European Union (EU) (grant agreement $n^{\circ}$ 603038). SE is supported as doctoral candidate by the Fund for Scientific Research Flanders (FWO). LD, RV and WJ are supported as clinical researchers by the FWO. RV is supported by an UZ Leuven starting grant. WAW is holder of the intermune Crystal Chair in interstitial lung diseases. JEM is supported by a fellowship from the ERS (RESPIRE2-2015-9192). SEV is sponsored by a postdoctoral grant from the FWO (FWO12G8715N). DSM and TSN received support from the EU 'Ideas' programme (ERC-2012-Stg 310898) and from the Flemish Scientific Fund (FWO G.0873.11.N.10)

\section{Availability of data and materials}

The datasets generated and/or analyzed during the current study are available from the corresponding author on reasonable request.

\section{Authors' contributions}

EJL and SE participated in lung tissue processing, performed DNA extractions, did all statistical analysis, interpreted the results and drafted the manuscript. DSM performed DNA extractions and all RTL measurements, and provided technical assistance in drafting the manuscript. SEV and JEM participated in lung tissue processing, performed all micro CT scans and measurements, and provided statistical assistance. AAB, RG and CHM performed additional experiments for revision of the manuscript. $A A B, R G$, $\mathrm{CHM}$ performed pilot experiments that contributed to the revision of the manuscript. LJDS, KM, AAB, RG, CHM, LJD, WAW, RV, GGR, NK, JCH, WJ and GMV contributed to and critically revised the manuscript. TSN, SEV, JEM and BMV participated in the design and coordination of the study, in the interpretation of the results and in drafting the manuscript. All authors have read and approved the manuscript.

\section{Ethics approval and consent to participate}

This study was approved by the local Ethics Committee (Medical Ethics Board of University Hospitals Leuven, Belgium; ML6385). According to Belgian law, organs from prospective donors, which are of insufficient quality for LTx and have been conclusively declined by the transplant surgeon, can be used for research purposes. All LTx patients gave written informed consent to use their lungs for research purposes.

\section{Competing interests}

The authors declare that they have no competing interests.

\section{Publisher's Note}

Springer Nature remains neutral with regard to jurisdictional claims in published maps and institutional affiliations.

\section{Author details}

${ }^{1}$ Laboratory of Respiratory Diseases, Department of Chronic Diseases, Metabolism \& Aging (CHROMETA), KU Leuven, Herestraat 49, O\&NI, box 706, B-3000 Leuven, Belgium. ${ }^{2}$ Centre for Environmental Sciences, Hasselt University, Hasselt, Belgium. ${ }^{3}$ Department of Pulmonology, St Antonius ILD Center of Excellence, St Antonius Hospital, Nieuwegein, the Netherlands. ${ }^{4}$ Department of Pathology, University Medical Center Utrecht, Utrecht, the Netherlands. ${ }^{5}$ Division of Heart and Lungs, University Medical Center Utrecht, Utrecht, the Netherlands. ${ }^{6}$ Department of Respiratory Diseases, University Hospitals Leuven, Leuven, Belgium. ${ }^{7}$ Section of Pulmonary, Critical Care, and Sleep Medicine, Yale University, New Haven, CT, USA. ${ }^{8}$ University of British Columbia James Hogg Research Centre, St. Paul's Hospital, Vancouver, BC, Canada. ${ }^{9}$ Department of Public Health \& Primary Care, KU Leuven, Leuven, Belgium.

Received: 29 September 2017 Accepted: 27 April 2018

Published online: 11 May 2018

References

1. López-Otín C, Blasco MA, Partridge L, Serrano M, Kroemer G. The hallmarks of aging. Cell. 2013;153(6):1194-217.

2. Lenart P, Krejci L. DNA, the central molecule of aging. Mutat Res. 2016;786: $1-7$.

3. Janssens JP, Pache JC, Nicod LP. Physiological changes in respiratory function associated with ageing. Eur Respir J. 1999:13(1):197-205.

4. Brandenberger C, Mühlfeld C. Mechanisms of lung aging. Cell Tissue Res. 2016;14:1-12.

5. Blackburn EH, Greider CW, Szostak JW. Telomeres and telomerase: the path from maize, Tetrahymena and yeast to human cancer and aging. Nat Med. 2006;12(10):1133-8.

6. Cawthon RM, Smith KR, O'Brien E, Sivatchenko A, Kerber RA. Association between telomere length in blood and mortality in people aged 60 years or older. Lancet Lond Engl. 2003;361(9355):393-5.

7. Aviv A. Genetics of leukocyte telomere length and its role in atherosclerosis. Mutat Res. 2012;730(1-2):68-74

8. Willeit P, Raschenberger J, Heydon EE, Tsimikas S, Haun M, Mayr A, et al. Leucocyte telomere length and risk of type 2 diabetes mellitus: new prospective cohort study and literature-based meta-analysis. PLoS One. 2014;9(11):e112483.

9. The Telomeres Mendelian Randomization Collaboration, Haycock PC, Burgess S, Nounu A, Zheng J, Okoli GN, et al. Association between telomere length and risk of Cancer and non-neoplastic diseases: a Mendelian randomization study. JAMA Oncol. 2017;3(5):636.

10. Steffens JP, Masi S, D'Aiuto F, Spolidorio LC. Telomere length and its relationship with chronic diseases - new perspectives for periodontal research. Arch Oral Biol. 2013;58(2):111-7.

11. Savale L, Chaouat A, Bastuji-Garin S, Marcos E, Boyer L, Maitre B, et al. Shortened telomeres in circulating leukocytes of patients with chronic obstructive pulmonary disease. Am J Respir Crit Care Med. 2009;179(7):566-71.

12. Snetselaar R, van Moorsel CHM, Kazemier KM, van der Vis JJ, Zanen P, van Oosterhout MFM, et al. Telomere length in interstitial lung diseases. Chest. 2015;148(4):1011-8.

13. Chilosi M, Carloni A, Rossi A, Poletti V. Premature lung aging and cellular senescence in the pathogenesis of idiopathic pulmonary fibrosis and COPD/emphysema. Transl Res J Lab Clin Med. 2013;162(3):156-73. 
14. Armanios M. Telomerase and idiopathic pulmonary fibrosis. Mutat Res Mol Mech Mutagen. 2012;730(1-2):52-8.

15. Stanley SE, Chen JJL, Podlevsky JD, Alder JK, Hansel NN, Mathias RA, et al. Telomerase mutations in smokers with severe emphysema. J Clin Invest. 2015;125(2):563-70.

16. Ley B, Newton CA, Arnould I, Elicker BM, Henry TS, Vittinghoff E, et al. The MUC5B promoter polymorphism and telomere length in patients with chronic hypersensitivity pneumonitis: an observational cohort-control study. Lancet Respir Med. 2017;5(8):639-647.

17. Rawlins EL, Hogan BLM. Epithelial stem cells of the lung: privileged few or opportunities for many? Dev Camb Engl. 2006;133(13):2455-65.

18. McDonough JE, Yuan R, Suzuki M, Seyednejad N, Elliott WM, Sanchez PG, et al. Small-airway obstruction and emphysema in chronic obstructive pulmonary disease. N Engl J Med. 2011;365(17):1567-75.

19. Verleden SE, Vasilescu DM, Willems S, Ruttens D, Vos R, Vandermeulen E, et al. The site and nature of airway obstruction after lung transplantation. Am J Respir Crit Care Med. 2014;189(3):292-300.

20. Martens DS, Plusquin M, Gyselaers W, De Vivo I, Nawrot TS. Maternal prepregnancy body mass index and newborn telomere length. BMC Med. 2016;14(1):148.

21. Levy MZ, Allsopp RC, Futcher AB, Greider CW, Harley CB. Telomere endreplication problem and cell aging. J Mol Biol. 1992;225(4):951-60.

22. Daniali L, Benetos A, Susser E, Kark JD, Labat C, Kimura M, et al. Telomeres shorten at equivalent rates in somatic tissues of adults. Nat Commun. 2013; 4:1597.

23. Armanios M. Telomeres and age-related disease: how telomere biology informs clinical paradigms. J Clin Invest. 2013;123(3):996-1002.

24. Gardner JP, Kimura M, Chai W, Durrani JF, Tchakmakjian L, Cao X, et al. Telomere dynamics in macaques and humans. J Gerontol A Biol Sci Med Sci. 2007;62(4):367-74

25. Alder JK, Barkauskas CE, Limjunyawong N, Stanley SE, Kembou F, Tuder RM, et al. Telomere dysfunction causes alveolar stem cell failure. Proc Natl Acad Sci U S A. 2015;112(16):5099-104.

26. Childs BG, Durik M, Baker DJ, van Deursen JM. Cellular senescence in aging and age-related disease: from mechanisms to therapy. Nat Med. 2015; 21(12):1424-35.

27. Faner R, Rojas M, Macnee W, Agustí A. Abnormal lung aging in chronic obstructive pulmonary disease and idiopathic pulmonary fibrosis. Am J Respir Crit Care Med. 2012;186(4):306-13.

28. Chen R, Zhang K, Chen H, Zhao X, Wang J, Li L, et al. Telomerase deficiency causes alveolar stem cell senescence-associated low-grade inflammation in lungs. J Biol Chem. 2015;290(52):30813-29.

29. Leslie KO, Cool CD, Sporn TA, Curran-Everett D, Steele MP, Brown KK, et al. Familial idiopathic interstitial pneumonia: histopathology and survival in 30 patients. Arch Pathol Lab Med. 2012;136(11):1366-76.

30. Fischer BM, Wong JK, Degan S, Kummarapurugu AB, Zheng S, Haridass $P$, et al. Increased expression of senescence markers in cystic fibrosis airways. Am J Physiol Lung Cell Mol Physiol. 2013;304(6):L394-400.

31. Lee J, Sandford AJ, Connett JE, Yan J, Mui T, Li Y, et al. The relationship between telomere length and mortality in chronic obstructive pulmonary disease (COPD). PLoS One. 2012;7(4):e35567.

32. Rode L, Bojesen SE, Weischer M, Vestbo J, Nordestgaard BG. Short telomere length, lung function and chronic obstructive pulmonary disease in 46,396 individuals. Thorax. 2013;68(5):429-35.

33. Tsuji T, Aoshiba K, Nagai A. Alveolar cell senescence in patients with pulmonary emphysema. Am J Respir Crit Care Med. 2006;174(8):886-93.

34. Birch J, Anderson RK, Correia-Melo C, Jurk D, Hewitt G, Marques FM, et al. DNA damage response at telomeres contributes to lung aging and chronic obstructive pulmonary disease. Am J Physiol Lung Cell Mol Physiol. 2015; 309(10):L1124-37.

35. Barnes PJ. Senescence in COPD and its comorbidities. Annu Rev Physiol. 2017;79:517-39

36. Courtwright AM, Fried S, Villalba JA, Moniodis A, Guleria I, Wood I, et al. Association of donor and recipient telomere length with clinical outcomes following lung transplantation. PLoS One. 2016;11(9):e0162409.

37. Faust HE, Golden JA, Rajalingam R, Wang AS, Green G, Hays SR, et al. Short lung transplant donor telomere length is associated with decreased CLADfree survival. Thorax. 2017;

38. Snetselaar R, van Batenburg AA, van Oosterhout MFM, Kazemier KM, Roothaan SM, Peeters T, et al. Short telomere length in IPF lung associates with fibrotic lesions and predicts survival. PLoS One. 2017;12(12):e0189467.
39. Verleden SE, de Jong PA, Ruttens D, Vandermeulen E, van Raemdonck DE, Verschakelen J, et al. Functional and computed tomographic evolution and survival of restrictive allograft syndrome after lung transplantation. J Heart Lung Transplant Off Publ Int Soc Heart Transplant. 2014;33(3):270-7.

40. Simanovsky N, Cohen-Cymberknoh M, Shoseyov D, Gileles-Hillel A, Wilschanski M, Kerem E, et al. Differences in the pattern of structural abnormalities on CT scan in patients with cystic fibrosis and pancreatic sufficiency or insufficiency. Chest. 2013;144(1):208-14.

41. Saferali A, Lee J, Sin DD, Rouhani FN, Brantly ML, Sandford AJ. Longer telomere length in COPD patients with a1-antitrypsin deficiency independent of lung function. PLoS One. 2014;9(4):e95600.

42. Montpetit AJ, Alhareeri AA, Montpetit M, Starkweather AR, Elmore LW, Filler $K$, et al. Telomere length: a review of methods for measurement. Nurs Res. 2014;63(4):289-99.

43. Aviv A, Hunt SC, Lin J, Cao X, Kimura M, Blackburn E. Impartial comparative analysis of measurement of leukocyte telomere length/DNA content by southern blots and qPCR. Nucleic Acids Res. 2011;39(20):e134.

44. Alder JK, Chen JJ-L, Lancaster L, Danoff S, Su S, Cogan JD, et al. Short telomeres are a risk factor for idiopathic pulmonary fibrosis. Proc Natl Acad Sci U S A. 2008;105(35):13051-6.

45. Quanjer PH, Tammeling GJ, Cotes JE, Pedersen OF, Peslin R, Yernault JC. Lung volumes and forced ventilatory flows. Eur Respir J. 1993;6(Suppl 16):5-40.

46. Quanjer PH, Stanojevic S, Cole TJ, Baur X, Hall GL, Culver BH, et al. Multiethnic reference values for spirometry for the 3-95-yr age range: the global lung function 2012 equations. Eur Respir J. 2012;40(6):1324-43.

47. American Thoracic Society. Single-breath carbon monoxide diffusing capacity (transfer factor). Recommendations for a standard technique-1995 update. Am J Respir Crit Care Med. 1995;152(6 Pt 1):2185-98.

\section{Ready to submit your research? Choose BMC and benefit from:}

- fast, convenient online submission

- thorough peer review by experienced researchers in your field

- rapid publication on acceptance

- support for research data, including large and complex data types

- gold Open Access which fosters wider collaboration and increased citations

- maximum visibility for your research: over $100 \mathrm{M}$ website views per year

At BMC, research is always in progress.

Learn more biomedcentral.com/submissions 